\title{
Respostas agudas pós-exercício dos níveis de lactato sanguíneo e creatinofosfoquinase de atletas adolescentes*
}

\author{
Carla Cristiane Silva1, Tamara Beres Lederer Goldberg², Renata Campos Capela ${ }^{3}$, Cilmery Suemi Kurokawa ${ }^{3}$,
} Altamir dos Santos Teixeira ${ }^{4}$, José Carlos Dalmas ${ }^{5}$ e Edílson Serpeloni Cyrino ${ }^{6}$

\section{RESUMO}

O crescente interesse de crianças e adolescentes por esportes competitivos induz a uma maior preocupação em prescrever treinamentos adequados a essa população específica. O conhecimento do impacto da intensidade de treinamento físico competitivo sobre a saúde de adolescentes são ainda incipientes na literatura científica. Este estudo objetivou investigar as respostas agudas do lactato sanguíneo (Lac) e da creatinofosfoquinase (CPK) após uma sessão de treinamento físico em atletas jovens treinados em diferentes modalidades esportivas. Participaram 43 adolescentes do sexo masculino entre 9 e 17 anos, distribuídos em três grupos: nadadores, tenistas, jogadores de futebol de salão. Os protocolos para cada modalidade seguiram o planejamento normal de uma sessão específica. A dieta no dia anterior à coleta foi padronizada e as 24 horas que precediam a avaliação foram dedicadas ao repouso dos atletas. Foram coletados $5 \mathrm{ml}$ de sangue da veia antecubital imediatamente antes da realização da sessão de treinamento, repetindo coleta idêntica, imediatamente após a sessão. Foi obtida a idade óssea para a avaliação da maturação esquelética pelo método de Greulich \& Pyle. Análise de variância KruskalWallis e teste U-de Mann-Whitney foram utilizados para comparações entre os grupos. Valores de $p<0,05$ foram considerados significantes. Em todas as modalidades esportivas estudadas os valores prévios do Lac e da CPK foram inferiores aos valores póssessão. Com relação aos três grupos de faixas etárias, tanto para o Lac como para a CPK, os valores na pré-sessão foram inferiores aos obtidos na pós-sessão, assinalando que para o grupo de 9 aos 11 anos, tanto na situação pré como na pós, os valores foram inferiores aos observados nos grupos de idade superior. Os resultados indicaram que a magnitude de aumento da CPK e do Lac

* Apoio da Fundação de Amparo à Pesquisa do Estado de São Paulo. FAPESP - Processo 04/07007-1.

1. Mestre em Pediatria da Faculdade de Medicina de Botucatu - UNESP.

2. Professora Adjunta Livre Docente do Departamento de Pediatria, Programa de Pós-graduação em Ginecologia, Obstetrícia e Mastologia da Faculdade de Medicina de Botucatu - UNESP, Disciplina de Medicina do Adolescente.

3. Pesquisadora do Centro de Pesquisa Clínica e Experimental em Pediatria do Departamento de Pediatria da Faculdade de Medicina de Botucatu - UNESP.

4. Professor Assistente do Departamento de Doenças Tropicais e Diagnóstico por Imagem da Faculdade de Medicina de Botucatu - UNESP.

5. Professor Associado do Departamento de Matemática Aplicada da Universidade Estadual de Londrina - UEL.

6. Grupo de Estudo e Pesquisa em Metabolismo, Nutrição e Exercício, Centro de Educação Física e Esporte - Universidade Estadual de Londrina.

Aceito em 13/6/07.

Endereço para correspondência: Profa. Tamara Beres Lederer Goldberg, Departamento de Pediatria, Disciplina de Medicina do Adolescente, Faculdade de Medicina de Botucatu - UNESP - 18607-918 - Botucatu, SP, Brasil. Tels.: (14) 3811-6274/3811-6083. E-mail: tamara@fmb.unesp.br
Palavras-chave: Treinamento físico. Adolescentes. Lactato e creatinofosfoquinase. Keywords: $\quad$ Physical practice. Adolescents. Lactate and creatine phosphokinase.

foram similares aos valores encontrados na literatura e demonstraram um aumento em função da faixa etária, indicando ser maturação biológica dependente.

\section{ABSTRACT}

\section{Acute post-exercise blood lactate and creatin phosphokinase levels responses in young athletes}

The growing interest of children and adolescents in competitive sports demands a higher concern with suitable training prescription to this specific population. The knowledge of the impact of the competitive physical training intensity over the young population's health is still scarce in the literature. Thus, the aim of this study was to examine acute responses in blood lactate (Lac) and creatine phosphokinase (CPK) concentration after a physical training session with young athletes trained in different sports modalities. Forty-three male adolescents (aged 9-17 years) were divided in three groups: swimmers, tennis players, and indoor soccer players. There were no changes in rules or procedures before or after the practice session. Diet and activity (no exercise) were standardized during the twenty-four hours before the evaluation. Venous blood samples ( $5 \mathrm{~mL}$ ) were collected from the antecubital vein immediately before and after the training session. The bone age was obtained through the Greulich \& Pyle method and was analyzed for skeleton maturity grading. Statistical analysis included Kruskal-Wallis variance analysis and Mann-Whitney $U$ test (alpha was set at 0.05). Both Lac and CPK were higher post-session than pre-session in all sports studied. Age appeared to influence Lac and CPK concentration. The age group between nine and eleven years old had lower values compared to the older group, both pre- and post-session. The increases in CPK and Lac with exercise are similar to the findings previously reported in the literature. Moreover, CPK and Lac are higher with older chronological age. Thus, it is possible to demonstrate that Lac and CPK have a positive correlation to bone maturation and chronological age.

\section{INTRODUÇÃO}

A mídia televisionada e escrita têm colocado em evidência vários jogadores profissionais de tênis, ginástica, natação, futebol e outros esportes em idades muito precoces, por volta dos 15 a 17 anos. Os excelentes resultados obtidos por atletas, ainda na fase infantil e durante a adolescência, em competições nacionais e internacionais de reconhecido impacto tem aumentado o interesse por atividades esportivas e resultante desta exposição, uma elevada proporção de crianças têm procurado os clubes de iniciação esportiva.

Embora, a Academia Americana de Pediatra(1) não recomende esforços vigorosos antes da maturação biológica completa de adolescentes, sabe-se que a especialização esportiva em idades mais 
jovens tem ocorrido graças à complexidade dos movimentos técnicos e ao alto grau de habilidade motora requisitados pelos esportes competitivos.

Alcançar níveis de excelência esportiva, independente da faixa etária é trabalho árduo e durante a infância e adolescência depende de muita cautela, em função do período exclusivo de crescimento físico e maturação biológica. Naughton et al.(2) destacam que o jovem atleta é normalmente exposto a altas cargas de treinamento concomitante às transformações biológicas, oriundas deste período da vida, aumentando substancialmente o risco de microtraumas e posterior interrupção do treinamento.

Com relação à prescrição do treinamento durante a idade infantil e adolescência tem-se relativamente pouco conhecimento, existe maiores discussões sobre "exercícios proibidos" em detrimento à adequada prescrição, principalmente relacionada à intensidade dos exercícios e aos períodos de recuperação entre as sessões de treinamento.

Entre os indicadores de intensidade do exercício físico, destaca-se o ácido lático, um intermediário do metabolismo dos carboidratos, principal metabólito do glicogênio em anaerobiose. Especificamente, durante os anos de crescimento físico e maturação biológica, especula-se que a magnitude do aumento deste marcador no sangue após exercício submáximo e máximo seja menor em crianças e adolescentes, quando comparada a de adultos ${ }^{(3-4)}$. Recentemente, Kaczor et al.(5) afirmaram que os mecanismos que envolvem a resposta da lactacidemia em crianças e adolescentes ainda não estão totalmente esclarecidos.

Outro aspecto de elevada importância relaciona-se ao período de recuperação entre as sessões de treinamento, pois, quando ocorrerem danos nos tecidos musculares, a enzima creatinofosfoquinase (CPK), com vasta distribuição no tecido contrátil, flui para a linfa, via interstício e entra na corrente sanguínea geral(6-7). A enzima CPK apresenta-se como exame laboratorial mais específico e provavelmente mais sensível para avaliação do dano muscular ${ }^{(8)}$. Embora essa enzima possa refletir alterações teciduais no miocárdio e no cérebro, representadas pela fração CPK-MB e CPK$\mathrm{BB}$ respectivamente, é resultante do tecido muscular esquelético (CPK-MM) que a CPK dosada no soro normal, representa. Isso ocorre devido à pequena massa de miocárdio e cérebro quando comparada com a massa muscular total(6). Assim, é provável que alta concentração dessa enzima no soro seja indicativa de lesão muscular, principalmente das células musculares, onde essa enzima está presente em altas proporções ${ }^{(9-10)}$.

Embora, exista muita preocupação entre os pesquisadores no sentido de não exceder os limites orgânicos de crianças e adolescentes vinculados a esportes competitivos, poucas são as investigações voltadas ao impacto da intensidade nas sessões de treinamento e os respectivos períodos para recuperação dos jovens atletas.

Neste sentido, o objetivo deste estudo foi investigar as respostas agudas imediatas do lactato sanguíneo e da creatinofosfoquinase de uma sessão de treinamento físico, de atletas jovens treinados em diferentes modalidades esportivas.

\section{MÉTODOS}

Participaram deste estudo 43 adolescentes do sexo masculino entre a faixa etária de 9 e 17 anos, subdivididos em três grupos: nadadores, tenistas, jogadores de futebol de salão. A pesquisa teve aprovação da Comissão de Ética em Pesquisa da Faculdade de Medicina de Botucatu-UNESP, protocolo-261/2004 em 10 de maio de 2004. Os adolescentes e seus responsáveis tiveram ciência do seu conteúdo, através de explanações, durante reuniões ocorridas no clube esportivo. Para que participassem da pesquisa, receberam e devolveram o termo de consentimento livre e esclarecido (TCLE) assinado pelo binômio adolescente e seu responsável.
Os adolescentes incluídos se declaravam voluntários, tinham experiência mínima de três anos no esporte específico, treinavam no mínimo por 10 horas semanais nos últimos seis meses, que antecederam a data de início da pesquisa, estavam vinculados a uma única modalidade esportiva e todos participavam de competições regionais e estaduais anualmente.

Como critérios de exclusão determinou-se que não participariam do estudo adolescentes tabagistas e/ou etilistas e aqueles que utilizassem qualquer suplementação nutricional e/ou fármacos ergogênicos. Também foram excluídos aqueles que apresentassem disfunções como, diabetes mellitus, endocrinopatias, puberdade precoce ou atrasada, deficiência de hormônio de crescimento e outras endocrinopatias.

Os adolescentes voluntários e que se enquadravam nos critérios de elegibilidade foram então convidados a comparecer ao Ambulatório de Adolescentes do Hospital das Clínicas da Faculdade de Medicina de Botucatu-UNESP, onde foi realizada uma entrevista com seus responsáveis, seguida de exame físico geral para que qualquer alteração física fosse detectada. Como os jovens encontravam-se em plena fase pubertária, optou-se pela avaliação da maturação esquelética como um indicador maturacional, já que a idade cronológica isolada é um indicador temporal muito limitado frente às modificações biológicas deste período da vida, as quais repercutem diretamente sobre o desempenho físico. Para avaliação da maturação esquelética foi utilizado raio-x de mão e punho esquerdo e a idade óssea foi obtida mediante o método de Greulich e Pyle(11)

Na seqüência os adolescentes foram encaminhados a uma clínica especializada, para realização da avaliação da composição corpórea total, realizada em uma unidade de densitometria óssea de dupla emissão com fontes de raio-x, utilizando um aparelho Hologic QDR 2000-Plus.

O desenho experimental foi fundamentado no treinamento diário proposto pelos treinadores, sem qualquer interferência dos pesquisadores, pois, o intuito foi de investigar a situação real de treinamento em detrimento à avaliação de um protocolo padronizado. Este modelo de delineamento, utilizado recentemente em outros estudos, tem sido utilizado envolvendo jovens vinculados a diferentes esportes competitivos ${ }^{(7,12-15)}$ na perspectiva de se observar o impacto da sessão e/ou jogo sobre diversas óticas, entre elas, os indicadores de estresse gerado pelo exercício.

Os protocolos seguiram o planejamento normal de uma sessão específica de acordo com cada modalidade e todos os adolescentes incluídos freqüentavam os treinamentos assiduidamente, sem qualquer intercorrência nos últimos seis meses que antecederam a pesquisa. Assim, o treinamento do tênis foi desenvolvido individualmente com o treinador e contemplava, em média, 50 minutos. Destes, os 10 minutos iniciais eram dedicados ao alongamento e golpes contra a parede. Na seqüência, era efetuado treinamento específico de jogo com movimentação em toda a quadra durante 30 a 35 minutos. Para finalizar o jovem atleta efetuava por volta de 10 minutos de jogadas paradas e alongamento final.

No protocolo da natação, os adolescentes alongavam fora da água por 5 minutos e posteriormente nadavam livre por mais 5 a 10 minutos. Na seqüência, o treinador acompanhava por aproximadamente 30 a 35 minutos o desempenho do nado crawl, impondo velocidade e cronometrando o tempo a cada 200 metros. Nos últimos 10 minutos, os jovens nadavam livre sem imposição de velocidade e por fim alongavam.

No treinamento de futebol de salão, cinco jovens efetuavam concomitantemente a sessão, que teve duração de 50 minutos. Para aquecimento os adolescentes realizavam corridas leves sem bola, durante 10 minutos. Na parte principal, o treinamento compunha-se de aproximadamente 30 a 35 minutos e exigia que os atletas realizassem jogadas com bola, movimentação rápida e finalização a meta. Por fim, os jovens executavam 10 minutos de alongamento. As respostas agudas imediatas foram mensuradas 
a partir destes modelos de protocolos específicos, sendo importante ressaltar que, em todas as sessões, os treinadores encorajavam os atletas particularmente com o aumento da velocidade em quadra e/ou piscina, além da técnica específica de cada esporte.

As vinte e quatro horas que precediam a avaliação da sessão de treinamento foram destinadas às orientações dietéticas do dia seguinte, bem como ao repouso pleno dos atletas para não ocorrerem inferências nos valores prévios dosados ${ }^{(8)}$. Nesta ocasião, os adolescentes receberam da nutricionista orientação dietética individual, totalmente adequada às suas necessidades orgânicas específicas e relacionadas a este período da vida, bem como ao esporte praticado. A dieta contemplava toda situação prévia ao treinamento, não sendo administrado qualquer alimento e/ou suplemento durante o treino. No próprio local foi realizada a coleta sanguínea pela equipe do Laboratório Experimental de Pediatria da Faculdade de Medicina de Botucatu-UNESP. Foram coletados $5 \mathrm{ml}$ de sangue da veia antecubital imediatamente antes da realização da sessão de treinamento, repetindo coleta idêntica imediatamente após a sessão de treinamento. O tempo de cada sessão foi devidamente cronometrado, assim como o tempo despendido entre o término do protocolo esportivo e o início da coleta sanguínea (tabela 1).

A amostra sanguínea foi coletada em tubo específico contendo EDTA e Fluoreto, e ainda nas dependências do clube foi imediatamente centrifugada e preparada para o congelamento $\left(-20^{\circ} \mathrm{C}\right)$, para posterior análise de lactato sanguíneo (Lac) como indicador de fadiga, e da creatinofosfoquinase total (CPK-total), indicador indireto de microlesões, ambos dosados a partir dos kits de química seca, analisador Vitros 950 (Ortho-Clinical Diagnostics, Rochester, NY, USA). Resumidamente, $10 \mu \mathrm{l}$ da amostra foi depositada sobre as lâminas com reativo seco o qual foi distribuído uniformemente pela camada de difusão para as camadas subjacentes. O lactado da amostra foi então oxidado pela lactato oxidase para piruvato e peróxido de hidrogênio. O peróxido de hidrogênio gerado oxidou o cromógeno 4-aminoantipirina e 1,7-dhidroxinaftaleno resultando em um complexo colorido. As amostras foram então incubadas e a densidade do reflexo do complexo colorido gerado foi medido por espectrofotometria, através do comprimento de onda de 540nm. A CPK baseou-se no mesmo princípio, sendo depositado $10 \mu$ l de amostra sobre as lâminas contendo N-acetilcisteína para ativar a CPK, sem necessidade de tratamento prévio da amostra. As amostras foram incubadas por 5 minutos a $37^{\circ} \mathrm{C}$ e após esta incubação, o peróxido de hidrogênio oxida o corante leuco para formar um corante e água. Assim, a taxa de alteração da densidade do reflexo monitorizada durante a incubação representa a atividade enzimática.

Os dados foram armazenados e analisados no programa Statistica versão VI. Análise de variância Kruskal-Wallis e teste U-de Mann-Witney foram efetuados para comparações entre os grupos, uma vez que através do teste Shapiro-Wilk verificou-se que a totalidade das variáveis não indicou distribuição normal dos dados. Os dados apresentados nos gráficos são dos valores médios \pm desvio-padrão. Foi considerado nível de significância estatística mínima quando $p<0,05$.

\section{RESULTADOS}

Indicadores de crescimento físico, de maturação esquelética e a composição corporal dos jovens atletas estão demonstrados na tabela 1. Entre os indicadores analisados não foi verificada diferença estatística entre os grupos para idade cronológica, idade óssea, peso, estatura, IMC e gordura relativa, demonstrando homogeneidade entre os adolescentes atletas que compunham os grupos. É importante ressaltar que a idade cronológica e a idade óssea foram similares entre os grupos esportivos, indicando que a maturação biológica refletiu o avançar dos anos cronológicos, entre os adolescentes inseridos neste estudo. Apenas no que se refere à massa corporal magra (MCM expressa em kg) observamse diferenças entre os grupos, quando os jovens vinculados ao futebol de salão apresentavam maior MCM que os nadadores e tenistas.

\section{TABELA 1}

Indicadores de crescimento, maturação esquelética e composição corporal de atletas adolescentes vinculados a diferentes modalidades esportivas

\begin{tabular}{lccc}
\hline \multicolumn{1}{c}{ Variáveis } & Tênis $(\mathbf{n}=\mathbf{1 0})$ & Natação $(\mathbf{n}=\mathbf{1 4})$ & Futsal (n = 19) \\
Idade cronológica (anos) & $14,1 \pm 2,0$ & $13,7 \pm 2,7$ & $13,9 \pm 1,8$ \\
Idade óssea (anos) & $14,1 \pm 2,8$ & $13,4 \pm 2,6$ & $14,2 \pm 2,7$ \\
Peso (kg) & $51,9 \pm 11,3$ & $51,8 \pm 11,5$ & $57,3 \pm 13,7$ \\
Estatura (m) & $1,64 \pm 0,12$ & $1,65 \pm 0,17$ & $1,65 \pm 0,13$ \\
IMC (kg/m $\left.{ }^{2}\right)$ & $18,8 \pm 1,7$ & $18,7 \pm 1,1$ & $20,6 \pm 2,7$ \\
MCM (kg) & $43,7 \pm 10,8$ & $43,2 \pm 11,4$ & $54,8 \pm 3,9 *$ \\
Gordura relativa (\%) & $17,6 \pm 5,6$ & $16,9 \pm 3,3$ & $14,8 \pm 4,6$ \\
\hline
\end{tabular}

$\overline{I M C}=$ índice de massa corporal; $\mathrm{MCM}=$ Massa corporal magra. Os resultados estão expressos em valores médios (DP). * Indica diferenças significantes com relação às outras modalidades $(\mathrm{p}<$ $0,05)$

A ingestão de energia e nutrientes consumidos anteriormente ao protocolo agudo de treinamento são apresentados na tabela 2. É possível observar que entre os indicadores nutricionais não foram observadas diferenças significantes. Esse resultado era esperado, em função da dieta e das recomendações nutricionais prévias ao treinamento terem sido similares entre os grupos, no sentido de minimizar o impacto nutricional frente aos indicadores bioquímicos.

A duração total da sessão de treinamento também não indicou diferenças significativas entre as modalidades, variando entre 48,3 e 51,6 minutos. Contudo, o intervalo entre o fim do protocolo e o início da coleta sanguínea diferiu entre as modalidades, demonstrando que na natação, o tempo despendido da coleta $(102,6 \pm$ 10,1 segundos) foi superior ao utilizado nas demais modalidades, $59,7 \pm 7,8$ segundos no tênis e 49,7 $\pm 12,2$ segundos no futsal), pois, a atividade aquática exige um intervalo específico entre secar a pele e realizar a veno punção.

\section{TABELA 2}

Ingestão de energia e nutrientes consumidos antes do protocolo agudo de treinamento de atletas adolescentes vinculados a diferentes modalidades esportivas

\section{Variáveis}

Tênis $(n=10) \quad$ Natação $(n=14) \quad$ Futsal $(n=19)$

Ingestão energética (kcal/kg) Proteína $\mathrm{g} / \mathrm{kg}$ $\mathrm{CHO} / \mathrm{kg}$ Lipídeos $\mathrm{g} / \mathrm{kg}$ Cálcio (mg/dia) Ferro (mg/dia) $\begin{aligned} 48,5 & \pm 11,5 \\ 1,9 & \pm 0,8 \\ 6,3 & \pm 1,4 \\ 1,7 & \pm 0,6 \\ 696,1 & \pm 430,7\end{aligned}$ $43,4 \pm 13,5$ $1,7 \pm 0,7$ $8,5 \pm 2,7$ $1,7 \pm 0,7$ $696,1 \pm 430,7 \quad 740,4 \pm 490,8$ $15,0 \pm 4,5 \quad 17,3 \pm 5,3$

Os resultados estão expressos em valores médios (DP).

Na figura 1 observa-se o comportamento do Lac e da CPK nas situações pré e pós-sessão de treinamento e entre as modalidades esportivas. Nota-se que todos os valores prévios foram inferiores aos valores pós-sessão, para todas as modalidades esportivas estudadas $(p<0,01)$. Entre as modalidades esportivas, as diferenças ocorreram entre os valores pré, com os resultados dos jogadores de futsal $(1,75 \pm 0,3 \mathrm{mmol})$, demonstrando valores superiores em relação aos demais esportes $(p<0,05)$, enquanto que na situação pós - sessão os valores dos nadadores foram superiores $(9,95 \pm 2,5 \mathrm{mmol})(\mathrm{p}<0,01)$. De forma similar, aos resultados do Lac, a CPK demonstrou-se significativamente aumentada no pós-exercício $(p<0,01)$. 


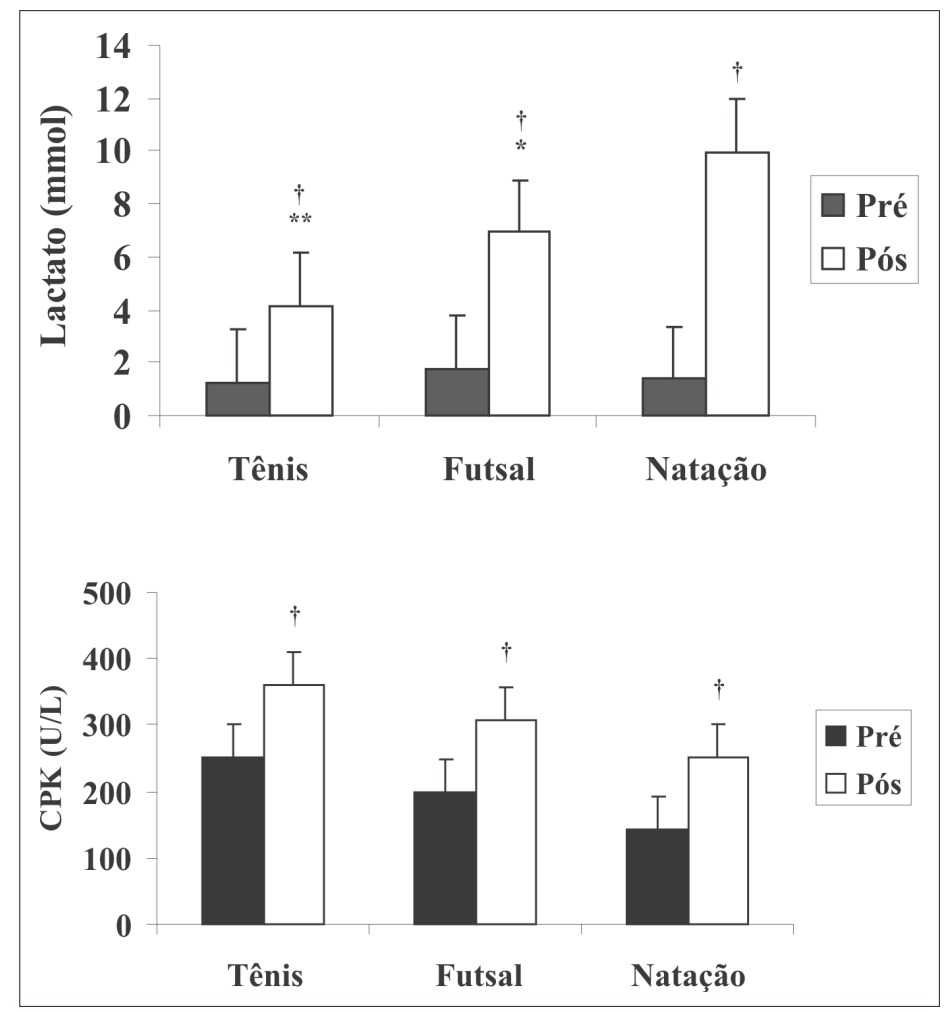

Figura 1 - Lactato e CPK nas situações pré e pós-sessão de treinamento nas diferentes modalidades esportivas.

$+p<0,01$ em comparação os valores pré- sessão de treinamento

${ }^{*} p<0,05 e{ }^{* *} p<0,01$ em comparação a outras modalidades

Na figura 2 observa-se o comportamento do Lac e da CPK nas situações pré e pós-sessão de treinamento e entre as faixas etárias agrupadas a partir da maturação esquelética, obtida pela realização da idade óssea. Na análise do Lac observa-se que em todos os grupos etários, os valores pós-sessão foram superiores aos valores pré $(p<0,01)$. Entre as faixas etárias, observa-se que nas idades de 9 a 11 anos os valores pós-exercício são inferiores $(4,0$ $\pm 2,3 \mathrm{mmol})$ aos das idades de 12 a $14(7,7 \pm 3,6 \mathrm{mmol})$ e 15 a 17 anos $(8,7 \pm 3,4 \mathrm{mmol})$.

Quanto aos valores da CPK, observa-se aumento tanto nos valores prévios como nos resultados obtidos pós-sessão de treinamento, em todas as faixas etárias, sendo os valores pós superiores aos valores pré-treinamento $(p<0,01)$. Entre os grupos etários verifica-se que dos 9 a 11 anos, tanto na situação pré (116,8 \pm $53,0 \mathrm{U} / \mathrm{L})$ como na pós-treinamento $(166,3 \pm 69,2 \mathrm{U} / \mathrm{L})$, os valores são inferiores aos observados nos grupos de idade mais avançada.

\section{DISCUSSÃO}

O presente estudo indicou aumento significativo nas concentrações de Lac pós-sessão de treinamento, em todas as modalidades esportivas estudadas ( $p<0,01$ ). Resultados similares foram encontrados, por Lima et al.(16), em estudo realizado com judocas adultos, com experiência de 10 anos no esporte, no que se refere ao comportamento do Lac. Os resultados dos judocas, na situação prévia à luta, foram de 2 a $3 \mathrm{mmol}$, aumentando para 10-12mmol pós-luta, sendo estes resultados significativamente superiores à média geral dos atletas adolescentes deste estudo $(7,23 \pm 3,78 \mathrm{mmol})$. Essas diferenças nas concentrações de Lac são assinaladas quando efetuam-se comparações entre crianças e adolescentes, em profunda fase de crescimento e desenvolvimento, com dados advindos de adultos, já completamente amadurecidos. Embora, as investigações sobre o impacto da treinabilidade, que contemplam os anos da infância e adolescência sejam
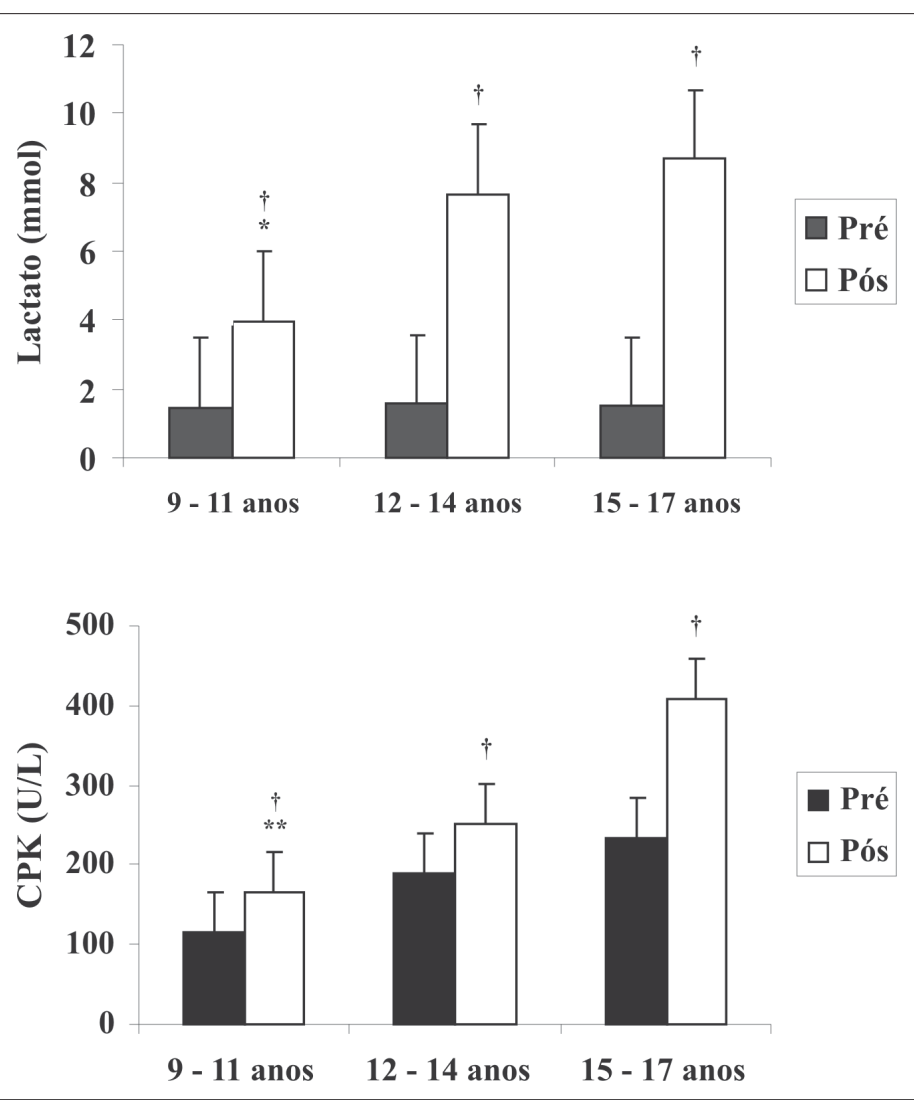

Figura 2 - Lactato e CPK nas situações pré e pós-sessão de treinamento nas diferentes faixas etárias (idade óssea)

$+p<0,01$ em comparação os valores pré-sessão de treinamento

** $p<0,01$ em comparação as demais faixas etárias

em pequeno número, sabe-se que em exercícios realizados em uma mesma intensidade, encontram-se concentrações menores de Lac sanguíneo em crianças, do que as obtidas em adultos ${ }^{3-5,17-}$ 18).

Corroborando com esses indicativos, Mahon et al. investigaram as concentrações de Lac entre crianças ( $n=9 ; 10,5 \pm 0,7$ anos) e adultos ( $n=9 ; 25,3 \pm 2,0$ anos) submetidos a um protocolo incremental em ciclo ergômetro. Os resultados do Lac após o exercício, realizado pelos adolescentes, demonstraram similaridade com o presente estudo e indicaram valores significativamente menores quando comparados aos dos adultos ${ }^{(17)}$.

No presente estudo, mais especificamente quanto aos resultados expressos na figura 2, observam-se diferenças entre as faixas etárias, quando estratificadas a partir da maturação esquelética. Os valores de Lac obtidos após as sessões de treinamento, entre os adolescentes mais jovens, foram significantemente inferiores aos apresentados pelas demais faixas etárias Foi evidenciado em estudo atual, abordando a atividade de marcadores enzimáticos anaeróbicos, provenientes de músculos esqueléticos que, adolescentes mais imaturos biologicamente, quando comparados com os seus pares, mais avançados na maturação, apresentavam atividade inferior de desidrogenase láctica $(\mathrm{DHL})^{(5)}$. Os resultados obtidos no presente estudo são consistentes e dependentes de uma menor capacidade anaeróbica, evidente em adolescentes, nos quais a puberdade está se iniciando.

Embora provenientes de uma pequena amostra, os dados obtidos apontam as concentrações de Lac após-exercício dependentes da idade óssea, ou seja, maturação biológica-dependente. Kaczor et al., através de revisão de literatura, recentemente demonstraram que, a idade exerce um forte impacto sobre a capacidade enzimática do músculo esquelético, particularmente durante o período de maturação biológica que envolve a transforma- 
ção do corpo infantil em corpo adulto(5). Vários mecanismos fisiológicos têm sido postulados na perspectiva de explicar as diferenças entre crianças, adolescentes e adultos e, provavelmente, os fatores envolvidos atuam de forma associada. Neste sentido, a literatura destaca: reduzida ação glicolítica expressa pela menor quantidade de fosfofrutoquinase (PFK) e lactato desidrogenase (DHL), como também pela elevada concentração de enzimas oxidativas, como a succinato desidrogenase (SDH), sugerindo que crianças e adolescentes são mais eficientes em obter energia através das vias oxidativas. Outro estudo suportou a hipótese de que crianças $(9,8 \pm 0,1$ anos) utilizam proporcionalmente mais gordura do que carboidratos quando comparados com adultos $(22,1 \pm 0,5$ anos) executando exercício prolongado, em mesma intensidade relativa ${ }^{(19)}$. Associado a esses fatores, pesquisadores indicam que os mais elevados estoques de lipídeos em crianças e adolescentes podem contribuir para minimizar a utilização de glicogênio durante o exercício físico ${ }^{(3-5,17,19)}$. Outros pesquisadores citam ainda as diferenças maturacionais na distribuição de fibras, com crianças apresentando maior composição de fibras tipo |(2,5,18,20-21). Para Ratel et al.(21) a associação dos fatores supracitados contribuem significativamente para explicar a considerável habilidade de crianças em resistir à fadiga, em protocolos intervalados de alta intensidade e curta duração, quando comparados com adultos.

Com relação aos valores de CPK, recentemente, Mujika et al.(10) citaram, a partir de revisão da literatura, que os valores de CPK podem ser utilizados como índice fisiológico de estresse induzido pelo exercício físico. Entre os fatores implicados na magnitude de aumento da CPK destacam-se, a duração e intensidade do exercício, além do nível individual de aptidão física do avaliado. Para Katirji e Al-jaberi(6), os níveis de CPK séricos tendem a diminuir em atletas treinados, como conseqüência de mecanismos adaptativos. Neste estudo, os adolescentes voluntários demonstraram experiência prévia nos esportes de, pelo menos, três anos, bem como, participação assídua no treinamento de seus respectivos esportes nos últimos seis meses, que antecederam a avaliação da sessão aguda. Se por um lado, acredita-se que indivíduos treinados cronicamente adquiram proteção contra o dano muscular (22), por outro, intervalos de recuperação diminuídos, podem gerar um estresse cumulativo que repercute em alterações bioquímicas, já evidenciadas na situação prévia ao treinamento(6,10).

Em outra investigação, as alterações agudas do treinamento de rugby de campo foram investigadas em atletas adultos $(n=25)$. Após 20 dias de treinamentos, seis horas por dia, a CPK indicou um aumento expressivo após o treinamento, sendo esse seis vezes maior do que os valores pré(22). Similarmente ao presente estudo, o que chamou mais a atenção não foi a magnitude do aumento da CPK no pós-exercício, ainda que significante, mas sim, foram os valores prévios, que se evidenciaram muito elevados, em média 116,8 \pm 53,0; 189,8 \pm 56,5 e 234,1 $\pm 158,9 \mathrm{U} / \mathrm{L}$, respectivamente para as faixas etárias de 9-11, 12-14 e 15-17 anos) uma vez que os valores de referência, considerados normais para esta enzima, estão entre 55 a $170(\mathrm{U} / \mathrm{L})$ para homens adultos ${ }^{(23)}$.

Embora a CPK indique importante variabilidade individual, estando atrelada ao nível de treinabilidade do atleta ${ }^{(6,10)}$, é relevante destacar o posicionamento de outros autores que, descrevem altos níveis séricos de CPK podendo ser potenciais indicadores de super-treinamento ${ }^{(9,24)}$. Os valores de CPK do presente estudo demonstraram variabilidade interindividual relativamente grande, entretanto, esses resultados não são exclusivos deste estudo, sendo partilhados por outros em suas investigações ${ }^{(10,18-19,21,25)}$.

$\mathrm{Na}$ perspectiva de verificar o impacto do intervalo de recuperação pós-exercício, Suzuki et al.(15) avaliaram 15 jogadores adultos de rugby. Após uma partida de rugby com 80 minutos de duração e 10 minutos de intervalo, os atletas foram submetidos a diferentes protocolos de recuperação. Um dos protocolos garantia que os atletas não efetuassem qualquer tipo de exercício sistematiza- do nas 48 horas posteriores ao jogo e no segundo protocolo, os atletas executaram uma hora de exercício de baixa intensidade na piscina após o jogo. Foram coletadas amostras sanguínea antes, após o jogo, 24 e 48 horas depois e, entre outros indicadores, os níveis de CPK foram dosados. Os resultados indicaram altos valores de CPK antes do jogo em ambos os grupos (média de 390U/ $\mathrm{L})$, seguido por significantes aumentos imediatamente após o jogo (482U/L). Contudo, o aumento mais expressivo ocorreu 24 horas após o término do jogo (680U/L), retornando aos valores pré-jogo, na coleta realizada após 48 horas $(400 \mathrm{U} / \mathrm{L})$. Os valores da CPK, ainda aumentados 24 horas após o término do jogo, corroboram com outros estudos da literatura ${ }^{(6,25,27)}$ onde se destaca o pico da CPK 24 horas após o treinamento físico. No presente estudo, não houve possibilidade de dosagens 24 e 48 horas após o fim das sessões de treinamento físico, em função dos procedimentos invasivos de coleta, particularmente quando são utilizados crianças e adolescentes, como sujeitos da pesquisa.

É importante destacar que Suzuki et al.(15) afirmaram, a partir de seus resultados, que o período de recuperação deve ser diretamente proporcional ao nível de intensidade exigido na sessão de treinamento e/ou jogo e, que valores prévios aumentados são indicativos de uma recuperação incompleta, podendo, inclusive, comprometer o desempenho físico. Neste sentido, parece existir grande importância do período de recuperação entre as sessões de treinamento, bem como, na fase que antecede competições importantes ${ }^{(10,28-29)}$. Mujika et al. ${ }^{(28)}$, em revisão da literatura entre 1982 e 2002, analisaram 11 investigações que destacavam os períodos de recuperação e magnitude nas concentrações da enzima CPK. Os resultados evidenciam que em 8 estudos, os níveis de CPK demonstraram-se significativamente reduzidos após intervalo e redução na intensidade dos treinamentos, indicando recuperação do estresse induzido pelo exercício com concomitante melhora na performance.

Os resultados do presente estudo indicaram similaridade aos encontrados na literatura específica, confirmando algumas hipóteses já aventadas, particularmente com relação à magnitude de aumento do Lac nas diferentes modalidades esportivas estudadas, indicando ser maturidade-dependente na situação pós-exercício.

Aparentemente, a concentração de Lac relacionada exclusivamente à idade cronológica, pode não ser um referencial fidedigno, quanto à intensidade, particularmente quando se objetiva reestruturar e/ou prescrever exercícios físicos para crianças e adolescentes em plena fase de maturação biológica.

Outro aspecto importante a ser discutido, resultante do presente estudo, foram os valores elevados de CPK na situação prévia à sessão de treinamento. As médias encontradas indicaram similaridade com outros estudos envolvendo adultos e neste aspecto, a literatura é pontual em assumir maiores períodos de recuperação após sessões e/ou jogos extenuantes, como estratégia de potencializar o desempenho(9,15,22-24). Entretanto, não foi relatada, pelos adolescentes deste estudo, qualquer queixa de cansaço excessivo e/ou dores musculares, o que denota preocupação, pois, aparentemente altas intensidades não indicaram necessariamente desgaste aumentado realizado pelos atletas, podendo estes facilmente ultrapassar os limites orgânicos.

Todos estes aspectos indicam dificuldade em se estabelecer níveis adequados de intensidade ao treinamento físico, particularmente durante a infância e os primeiros anos da adolescência. Assim, é prudente ter cautela na prescrição de exercícios, apoiando-se em vários indicadores, como os aspectos do crescimento, o amadurecimento biológico, a treinabilidade prévia adquirida, o esforço percebido durante as sessões e as metas a serem alcançadas, na perspectiva de potencializar as capacidades físicas envolvidas no esporte e minimizar quaisquer comprometimentos à saúde. 


\section{AGRADECIMENTOS}

Márcia Sayuri Takaasi - Ortho-Clinical Diagnostics - Johnson \& Johnson Produtos Profissionais pelo apoio com as técnicas e dosagens efetuadas e a llana Lederer Goldberg, pela versão do resumo em inglês.

Todos os autores declararam não haver qualquer potencial conflito de interesses referente a este artigo.

\section{REFERÊNCIAS}

1. Anderson SJ, Griesemer BA, Johnson MD, et al. Intensive training and sports specialization in young athletes. Am Acad Pediatr, Comm Sports Med Fit. 2000; 106:154-7

2. Naugthon G, Farpour-Lambert NJ, Carlson J, Bradney M, Praagh EV. Physiology issues surrounding the performance of adolescent athletes. Sports Med. 2000; 30:309-25.

3. Denadai BS, Greco CC, Donega MR. Comparação entre a velocidade de limiar anaeróbio e a velocidade crítica em nadadores com idade de 10 a 15 anos. Rev Paul Educ Fís. 1997;11:128-33.

4. Greco CC, Denadai BS, Pellegrinotti IL, Freitas AB, Gomide E. Limiar anaeróbico e velocidade crítica determinada com diferentes distâncias em nadadores de 10 a 15 anos: relação com a performance e a resposta do lactato sanguíneo em testes de endurance. Rev Bras Med Esporte. 2003;9:2-8.

5. Kazcor JJ, Ziolkowski W, Popinigis J, Tarnoploski MA. Anaerobic and aerobic enzyme activities in human skeletal muscle from children and adults. Pediatric Research. 2005;57:331-5.

6. Katirji B, Al-Jaberi MM. Creatine kinase revisited. J Clin Neuromuscular Disease. $2001 ; 2: 158-63$.

7. Mashiko T, Umeda T, Nakaji S, Sugawara K. Position related analysis of the appearance of and relationship between post-match physical and mental fatigue in university rugby football players. Br J Sports Med. 2004;38:617-21.

8. Picarelli MM, Kaise GRRF, Mühlen CAV. Dosagem laboratorial de enzimas musculares e diagnóstico equivocado de polimiosite juvenil: problemas na avaliação clínica e na fase pré-analítica. Rev Bras Reumatol. 2004;44:224-6.

9. Brenner IK, Natale VM, Vasilion P, Moldovenu A, Shek PN, Shepard RJ. Impact of three different types of exercise on components of the inflammatory response. Eur J Appl Physiol. 1999;80:452-60

10. Mujika I, Padilla S, Pyne D, Busso T. Physiological changes associated with the pre-event taper in athletes. Sports Med. 2004;34:891-927.

11. Greulich WW, Pyle SI. Radiographic atlas of skeletal development of the hand and wrist. $2^{\text {nd }}$ ed. Standford: University Press; 1959.

12. Lima F, Falco V, Baima J, Carazzato JG, Pereira RMR. Effect of impact load and active load on bone metabolism and body composition of adolescent athletes. Med Sci Sports Exerc. 2001;33:1318-23
13. Scheett TP, Nemet D, Stoppani J, Maresh CM, Newcomb R, Cooper DM. The effect of endurance-type exercise training on growth mediators and inflammatory cytokines in pre-pubertal and early pubertal males. Pediatrics Research. 2002;52:491-7.

14. Nemet D, Rose-Gottron CM, Mills PJ, Cooper DM. Effect of water polo practice on cytokines, growth mediadors, and leukocytes in girls. Med Sci Sports Exerc. 2003;35:356-63.

15. Suzuki M, Umeda T, Nakaji S, Shimoyama T, Mashiko T, Sugawara K. Effect of incorporating low intensity exercise into the recovery period after a rugby match. Br J Sports Med. 2004;38:436-40.

16. Lima EV, Tortoza C, Rosa LCL, Martins RABL. Estudo da correlação entre a velocidade de reação motora e o lactato sanguíneo, em diferentes tempos de luta no judô. Rev Bras Med Esporte. 2004;10:339-43.

17. Mahon AD, Duncan GE, Howe CA, Corral PD. Blood lactate and perceived exertion relative to ventilatory threshold: boys versus men. Med Sci Sports Exerc. 1997;29:1332-7.

18. Boisseau N, Delamarche P. Metabolic and hormonal responses to exercise in children and adolescents. Sports Med. 2000;30:405-22.

19. Timmons BW, Bar-Or O, Riddell MC. Oxidation rate of exogenous carbohydrate during exercise is higher in boys than in men. J Appl Physiol. 2003;94:278-84.

20. Armstrong N, Welsman JR, Chia MYH. Short term power output in relation to growth and maturation. Br J Sports Med. 2001;35:118-24.

21. Ratel S, Lazaar N, Williams CA, Bedu1 M, Duche' P. Age differences in human skeletal muscle fatigue during high-intensity intermittent exercise. Acta Paediatr. 2003;92:1248-54.

22. Mashiko T, Umeda T, Nakaji S, Sugawara K. Effects of exercise on the physical condition of college rugby players during summer training camp. Br J Sports Med. 2004;38:186-90.

23. Schrama YC, Hené RJ, De Jonge N, Joles JA, Van Rijn HJM, Bär DR, et al. Efficacy and muscle safety of fluvastatin in cyclosporine-treated cardiac and renal transplant recipients: an exercise provocation test. Transplation. 1998;66: 1175-81.

24. Halson SL, Jeukendrup AE. Does overtraining exist? An analysis of overtraining research. Sports Med. 2004;34:967-81.

25. Shave R, Dawson E, Whyte G, George K, Gaze D, Collinson P. Altered cardiac function and minimal cardiac damage during prolonged exercise. Med Sci Sports Exerc. 2004;36:1098-103.

26. Achour Júnior A, Borges PSS. Alongamento: efeitos na dor muscular tardia e níveis de creatinaquinase. Estudo de caso usando-se regressão linear múltipla e redes neurais artificiais. Rev Bras Ativ Física Saúde. 1997;2:24-33.

27. Child RB, Wilkinson DM, Folowfield JL. Effects of training taper on tissue damage indices, serum antioxidant capacity and half-marathon running performance. Int J Sports Med. 2000;21:325-31.

28. Mujika I, Padilla S. Scientific bases for precompetition tapering strategies. Med Sci Sports Exerc. 2003;35:1182-7. 